\title{
On the Relationship between ANOVA Main Effects and Average Treatment Effects
}

\author{
Linda Graefe ${ }^{1}$, Sonja Hahn ${ }^{2}$, and Axel Mayer ${ }^{3}$ \\ ${ }^{1}$ Friedrich Schiller University Jena, ORCID: 0000-0003-0368-667X \\ ${ }^{2}$ University of Education Karlsruhe, ORCID: 0000-0002-5461-6383 \\ ${ }^{3}$ Bielefeld University, ORCID: 0000-0001-9716-878X
}

This article has been accepted for publication in Multivariate Behavioral Research, published by Taylor and Francis.

April 1, 2022

\begin{abstract}
Author Note
Correspondence concerning this article should be addressed to Linda Graefe, Department of Psychological Methods, Institute of Psychology, Friedrich Schiller University, Am Steiger 3, Haus 1, D-07743 Jena, Germany, e-mail: linda.graefe@uni-jena.de

This work was supported by a grant to the last author from the German Research Foundation (DFG; Grant No. MA 7702/1-1 and MA 7702/1-2).
\end{abstract}




\begin{abstract}
We adopt a causal inference perspective to shed light into which ANOVA type of sums of squares (SS) should be used for testing main effects and whether main effects should be considered at all in the presence of interactions. We consider balanced, proportional and nonorthogonal designs, and models with and without interactions. When the design is balanced, we show that the average treatment effect is estimated by the main effects obtained by type I, II, and III sums of squares. In proportional designs, we show that the average treatment effect is estimated by the the type I and type II main effects, whereas type III SS yield biased estimates of the average treatment effect if there are interactions. When the design is nonorthogonal, ANOVA type I is always highly biased and ANOVA type II and III main effects are biased if there are interactions. We include a simulation study to illustrate the magnitude of the bias in estimating the average treatment effect across a variety of conditions, and provide recommendations for applied researchers.

Keywords: Average causal effect, Average treatment effect, Main effect, ANOVA, Analysis of variance, Sums of squares.
\end{abstract}




\section{On the Relationship between ANOVA Main Effects and Average Treatment Effects}

\section{Introduction}

Multifactorial between-subject designs are widespread in the social and behavioral sciences. In order to evaluate (main) effects of treatments or experimental manipulations, researchers often rely on analysis of variance (ANOVA) having causal interpretations in mind. This notion can be traced back to Fisher $(1925 / 1973)$ who introduced ANOVA procedures in terms of causes: "A type of data, which is of very common occurrence, may be [...] treated by the analysis of variance, that is by separation of the variance ascribable to one group of causes from the variance ascribable to other groups" (Fisher, 1925/1973, p. 214). Many review articles demonstrate that ANOVA is one of the most popular statistical approaches (Bangert \& Baumberger, 2005; Elmore \& Woehlke, 1998; Keselman et al., 1998; Lindstromberg, 2016, Skidmore \& Thompson, 2010; Warne et al., 2012; Yoo et al., 2015). Given its popularity, it is surprising that there is no consensus on how to apply ANOVA techniques. According to the terminology introduced by the software program SAS (SAS, 2004), type I, II, and III sums of squares are distinguished from each other in designs without empty cells. ANOVA type IV can be applied in designs with empty cells, but such designs are not the focus of this article. The ANOVA types differ with regard to the null hypothesis tested (see Maxwell et al., 2018, for an overview). However, the choice of different ANOVA types seems to depend more on the default settings of popular statistical software (see Table 1 for some examples) than on the well-informed decision of the researchers (Navarro, 2015, p. 542).

Taking a well-informed decision is even harder, as there are several recommendations in the literature favoring type I (e.g., Chambers \& Hastie, 1991; Overall \& Spiegel, 1969), type II (e.g., Fox, 2016; Langsrud, 2003; Nelder, 1976, 1977; Overall \& Spiegel, 1969), or type III (e.g., Howell, 2013; Shaw \& Mitchell-Olds, 1993; Speed et al., 1978) or advising against type I (e.g., Fox, 2016; Searle, 1987), type II (e.g., Overall et al., 1975), or type III (e.g., Langsrud, 2003; Nelder, 1994; Nelder \& Lane, 1995; Venables, 1998, 2003). The discussion concerns topics such as computational issues, sum of squares, marginality assumptions, interpretation of results, power, 


\section{Table 1}

Default setting of ANOVA types for different statistical computer programs

\begin{tabular}{ll}
\hline Program (Version) & Default Setting \\
\hline Genstat (6) & Type I \\
Mathematica (11) & Type I \\
R: anova in stats (4.0.2) & Type I \\
S+ (8.2) & Type I \\
\hline R: Anova in car (3.0-12) & Type II \\
\hline JMP (13.1) & Type III \\
Minitab Express (1.5) & Type III \\
Prism (7) & Type III \\
R: afex (1.0-1) & Type III \\
SPSS (26) & Type III \\
STATA (16) & Type III \\
SYSTAT (13) & Type III \\
\hline SAS (9.4) & Type I + III \\
Statistica (13.1) & Type III + Type IV \\
\hline
\end{tabular}

or the sequence of factors considered. For a summary of the controversy see, for example, Hector et al. (2010), Herr and Gaebelein (1978), or Macnaughton (1998).

Another controversy exists, as several authors advise against the interpretation of ANOVA main effects in the presence of significant interactions (e.g., Appelbaum \& Cramer, 1974, p. 340, Games, 1973, p. 305, Kirk, 2013, p. 367, Meyer, 1991, p. 572). Nevertheless, many researchers are in fact interested in average effects of treatments in addition to learning about interactions (Burdick, 1983; Elston \& Bush, 1964; Mayer \& Thoemmes, 2019; Tukey, 1977). This is supported by causal inference literature. There, it is typically assumed that there are interindividual differences in treatment effects and their average is a meaningful quantity (see, e.g., Imbens \& Rubin, 2015, p. 36). Thus, we argue that it is still worthwhile to ask research 
questions regarding main or average treatment effects (ATE) in the presence of interactions between treatment variable and covariate(s), as there are situations where they are appropriate and meaningful (see the discussion for two examples).

While all three ANOVA types lead to identical results in balanced designs with equal cell frequencies, there can be considerable differences in unbalanced designs which are common in the social and behavioral sciences. Keselman et al. (1998) report that $72 \%$ of factorial research designs in the educational sciences are unbalanced. The key difference between the three ANOVA types lies in the definition of the main effect and therefore in the hypothesis tested. The question of "How to define a main effect in a nonorthogonal ANOVA?" still lacks a universal answer. In contrast to the somewhat obscured definitions of main effects in the ANOVA literature, the causal inference literature (e.g., Neyman, 1923/1990; Pearl, 2009; Rubin, 2005; Steyer et al., 2000) provides us with a clear and unambiguous definition of an average treatment effect. In this literature, the average treatment effect is defined as the unconditional expectation of the individual treatment effects. In addition, theories of causal effects are very explicit in formulating causality conditions under which average treatment effects can be interpreted as causal effects (see, e.g., Steyer et al., 2014). Throughout this paper we assume that at least one of the causality conditions holds. Thus, unbiasedness (Steyer et al., 2014) or strong ignorability (Rosenbaum \& Rubin, 1983) is implied and the average treatment effect can be interpreted causally as average causal effect.

In this paper, we use arguments and definitions from the causal inference literature to clarify the meaning of different types of ANOVA main effects and to aid researchers in the choice of which ANOVA type is best suited to answer their specific research question. We show in which designs and under which conditions the ANOVA main effects correspond to average treatment effects and thus have a causal interpretation. We consider balanced, proportional and nonorthogonal designs, and models with and without interactions. In a simulation study, we confirm our theoretical results and examine the severity of bias under different conditions. 


\section{Main and Average Effects}

In order to define main and average treatment effects in factorial designs, we use the following notation: Let $X$ be the categorical treatment variable with values $x=0,1, \ldots, p$, let $K$ be a categorical covariate with values $k=0,1, \ldots, j$, and let $Y$ be a continuous outcome variable. In this article we focus on tests for the average treatment effect of $X$ on $Y$ possibly accounting for $K$. This setting is very general and encompasses many factorial designs, because $K$ can also represent a combination of values of multiple categorical covariates (e.g., Wüthrich-Martone, 2001, chap. 6). We restrict ourselves to designs without continuous covariates in our presentation but the reasoning generalizes to continuous covariates as well. For didactic reasons, we use two different ways to define main and average treatment effects, namely in terms of effect computation and in terms of model comparisons. An example at the end of this section illustrates the meaning of the mathematical terms and equations.

\section{Definitions of Main Effects and Average Treatment Effects Using Effect Computations}

Main effects $(M E)$ and average treatment effects $(A T E)$ can be defined via effect computations based on cell means originating from the literature on hypothesis tests (e.g., Herr \& Gaebelein, 1978). For this purpose, treatment conditions $X=x$ and $X=x^{\prime}$ with $x \neq x^{\prime}$ are compared to each other using differences of sums of weighted cell means. The subscripts $x x^{\prime}$ on $M E$ indicate the two treatment groups which are compared to each other. The superscript indicates that the main effect is based on ANOVA type I, II, or III. As we focus on research designs with representative samples and stochastic group sizes, the theoretical weights are introduced as probabilities. For empirical applications, probabilities are estimated using corresponding frequencies 1

\footnotetext{
${ }^{1}$ While the causal inference literature and the related calculation of the ATE uses probabilities, ANOVA approaches as part of the general linear model family - assume the factors (and their distributions) to be fixed and often use frequencies as weights. As our focus is on potentially causally interpretable treatment effects and our descriptions of effects are rather theoretical than empirical, we will focus on probabilities subsequently. Nevertheless, theoretical probabilities are strongly related to empirically observed frequencies: Relative frequencies are used as point estimates of probabilities.
} 


$$
\begin{aligned}
M E_{x x^{\prime}}^{\mathrm{I}}= & \sum_{k=0}^{j} E(Y \mid X=x, K=k) \cdot P(K=k \mid X=x)-\sum_{k=0}^{j} E\left(Y \mid X=x^{\prime}, K=k\right) \cdot P\left(K=k \mid X=x^{\prime}\right) \\
M E_{x x^{\prime}}^{\mathrm{II}}=\sum_{k=0}^{j} Q^{i f}(Y \mid X=x, K=k) \cdot \frac{1}{j+1} & -\sum_{k=0}^{j} Q^{i f}\left(Y \mid X=x^{\prime}, K=k\right) \cdot \frac{1}{j+1} \\
M E_{x x^{\prime}}^{\mathrm{III}}=\sum_{k=0}^{j} E(Y \mid X=x, K=k) \cdot \frac{1}{j+1} & -\sum_{k=0}^{j} E\left(Y \mid X=x^{\prime}, K=k\right) \cdot \frac{1}{j+1} \\
A T E_{x x^{\prime}}=\sum_{k=0}^{j} E(Y \mid X=x, K=k) \cdot P(K=k) & -\sum_{k=0}^{j} E\left(Y \mid X=x^{\prime}, K=k\right) \cdot P(K=k)
\end{aligned}
$$

Type I main effects $\left(M E_{x x^{\prime}}^{\mathrm{I}}\right)$ compare sums of the group specific cell means $E(Y \mid X=x, K=k)$ weighted by the conditional probability $P(K=k \mid X=x)$. The difference can be simplified (see, e.g., Steyer \& Nagel, 2017, p. 294) and also be written as $M E_{x x^{\prime}}^{\mathrm{I}}=E(Y \mid X=x)-E\left(Y \mid X=x^{\prime}\right)$. Thus, type I main effects ignore $K$ altogether and correspond to the main effect of $X$ in a model where $X$ is entered first (see the definitions using model comparisons below for sensitivity of ANOVA main effects to the sequence of terms).

Type II main effects $\left(M E_{x x^{\prime}}^{\mathrm{II}}\right)$ use interaction free (if) cell means $Q^{i f}(Y \mid X=x, K=k)$ gained by the linear quasi-regression $Y \sim 1+X+K$ (notation based on Wilkinson and Rogers (1973)). As the quasi-regression $Q^{i f}(Y \mid X, K)$ ignores possible interaction terms, its values may differ to the ones of the genuine regression $E(Y \mid X, K)$ and is therefore only called quasi-regression. Steyer and Nagel (2017, p. 225 ff.) provide detailed information on that topic. The interaction free cell means are then divided by the total number of covariate values $j+1$. An alternative way of computation of type II main effects specific for $2 \times 2$ designs using $E(Y \mid X=x, K=k)$ instead of $Q^{i f}(Y \mid X=x, K=k)$ is shown in Appendix A (see supplemental materials at https://osf.io/vjda8).

Type III main effects $\left(M E_{x x^{\prime}}^{\mathrm{III}}\right)$ are sums of the group specific cell means divided by the total number of covariate values $j+1$. That is, every cell mean is weighted by the same value assuming a uniformly distributed covariate. In contrast to ANOVA type II, this computation 
accounts for possible interactions. If there is no interaction, the main effects of type III ANOVA equal the ones of type II.

Average treatment effects $\left(A T E_{x x^{\prime}}\right)$ are sums of the group specific cell means weighted by the unconditional probability $P(K=k)^{2}$. That means, we average the differences of two treatment conditions over the marginal distribution of $K$.

As the definitions show, ANOVA main effects and average treatment effects differ in the cell means used as well as in the weighting of these means. Their equivalence will be crucial in order to show when main and average effects correspond to each other.

\section{Definitions of Main Effects and Average Treatment Effects Using Model Comparisons}

Another definition of main effects and average treatment effects of $X$ is most easily described using the notation introduced by Wilkinson and Rogers (1973). Furthermore, the adequate coding is crucial for the regression coefficients to correspond to the definitions of main and average treatment effects. This is equivalent to the selection of adequate contrasts of the predictor variables. Different coding schemes imply orthogonal or nonorthogonal contrasts and may influence the model parameters as well as the computation of sums of squares (SS) and, thus, the results of statistical tests for significance of predictors. While the coding does not influence the statistical tests of ANOVA type I and II main effects, type III is sensitive to the coding scheme applied (Langsrud, 2003; Navarro, 2015). Regarding model comparisons, ANOVA type III only leads to correct results (in terms of ANOVA type III definition) if orthogonal coding is used. There are different ways, specifying such orthogonal contrasts, such as effect coding, (reverse) Helmert coding, or orthogonal polynomial coding (see, e.g., Pedhazur \& Schmelkin, 1991). For definitions of ANOVA main effects we assume orthogonal coding, such as effect coding (eff), for $X$ and $K$ for all types of ANOVA main effects. That is, the regression coefficients represent deviations from the intercept. The intercept itself represents the mean of all group means. All

\footnotetext{
${ }^{2}$ This computation corresponds exactly to Robin's g-formula without time-varying variables, an alternative approach for causal inference often used in epidemiology (for more details on g-methods see, for example, Hernán \& Robins, 2020, Naimi et al., 2016, Robins, 1986).
} 
other orthogonal coding schemes would be adequate, too. For the ATE we assume dummy coding (dum) for $X$ and mean centered dummy coding (cen) for $K$. That is, for all values of $X$ and $K$ indicator variables are used (except the reference group). Additionally, the indicator variables of covariate(s) are centered around their own mean. This coding allows for directly interpreting the regression coefficient of $X$ as treatment effect compared to a reference group. All presented coding schemes are exemplified in Appendix B (see supplemental materials at https://osf.io/vjda8). The different effects are then defined via the following model comparisons:

$$
\begin{array}{llll}
M E^{\mathrm{I}}: & Y \sim 1+X^{\mathrm{eff}} & \text { vs. } & Y \sim 1 \\
M E^{\mathrm{II}:} & Y \sim 1+X^{\mathrm{eff}}+K^{\mathrm{eff}} & \text { vs. } & Y \sim 1+K^{\mathrm{eff}} \\
M E^{\mathrm{III}}: & Y \sim 1+X^{\mathrm{eff}}+K^{\mathrm{eff}}+X^{\mathrm{eff}} \cdot K^{\mathrm{eff}} & \text { vs. } & Y \sim 1+K^{\mathrm{eff}}+X^{\mathrm{eff}} \cdot K^{\mathrm{eff}} \\
A T E: & Y \sim 1+X^{\mathrm{dum}}+K^{\mathrm{cen}}+X^{\mathrm{dum}} \cdot K^{\mathrm{cen}} & \text { vs. } & Y \sim 1+K^{\mathrm{cen}}+X^{\mathrm{dum}} \cdot K^{\mathrm{cen}}
\end{array}
$$

The test for ANOVA type I main effects $\left(M E^{\mathrm{I}}\right)$ compares a model with one factor to a model without any factors. Type I main effects are sensitive to the sequence of terms and vary with the ordering of the factors included (see, e.g., Herr \& Gaebelein, 1978). Recall that we are interested in the effect of $X$ and, thus, show the definition when $X$ is entered first. When (instead of $X) K$ is entered first, the type I main effect of $X$ corresponds to the type II main effect.

Type II main effects $\left(M E^{\mathrm{II}}\right)$ are tested via the comparison of a model with $X$ and $K$ to a model with only $K$. In contrast to type I main effects, type II main effects are not sensitive to the sequence of terms. They follow the principle of marginality and always ignore higher-order terms for the tests of lower-order terms. For this reason, proponents of this approach argue that the interpretation of main effects is only legitimate without significant interaction terms (e.g., Appelbaum \& Cramer, 1974).

Testing for ANOVA main effects type III $\left(M E^{\mathrm{III}}\right)$, a model with all terms included is compared to a model without the main effect of $X$ (but - in contrast to type II - with interaction). If there is no interaction, the main effects of type III equal those of ANOVA type II. Type III main effects are sensitive to the coding scheme for $X$. As mentioned above we assume an orthogonal coding (e.g., effect coding) for all factors. Type III tests account for higher-order terms when 


\section{Table 2}

Nonorthogonal $2 \times 2$ design example: Population means $E(Y \mid X=x, K=k)$, corresponding probabilities $P(X=x, K=k)$ and their marginals $P(X=x)$ and $P(K=k)$ in italics

\begin{tabular}{rrrrrr}
\hline & \multicolumn{5}{c}{ Sex } \\
\cline { 2 - 5 } Treatment & Women $(K=0)$ & Men $(K=1)$ & $P(X=x)$ \\
\hline Control $(X=0)$ & 85 & 0.35 & 110 & 0.15 & 0.50 \\
Cognitive Behavioral Therapy $(X=1)$ & 76 & 0.05 & 116 & 0.45 & 0.50 \\
\hline$P(K=k)$ & & 0.40 & & 0.60 & \\
\hline
\end{tabular}

testing lower-order terms.

At first glance, the average treatment effect test looks identical to the type III test.

However, in order for this model comparison to correspond to the average effect, we have to use mean centered dummy coding for $K$ instead of effect coding. As this is most common for these models, we use dummy coding for $X$ but other codings would work, too, since we are only interested in the average effect of $X$. R code (R Core Team, 2020) for all these model comparisons and the standard ANOVA output for a simple example can be found at https://osf.io/vjda8 so that the interested reader can reproduce the theoretical results.

\section{Minimal $2 \times 2$ Example}

In order to illustrate the definitions of main effects and average treatment effects, we use the minimal example of an unbalanced $2 \times 2$ between-subjects design. This nonorthogonal design has the advantage that all main and average effects differ, making it easier to illustrate the differences between the approaches. $X$ is a binary treatment variable with values $X=0$ (control group) and $X=1$ (cognitive behavioral therapy (CBT)). $K$ is a categorical covariate sex with values $K=0$ (women) and $K=1$ (men). The continuous outcome variable depression is denoted by $Y$ with higher values representing higher depression. Table 2 shows the population cell means $E(Y \mid X=x, K=k)$ and the cell probabilities $P(X=x, K=k)$ as well as the corresponding marginal probabilities $P(X=x)$ and $P(K=k)$. 
Following the definitions introduced above, the ANOVA type I main effect in our example is:

$$
\begin{aligned}
M E_{10}^{\mathrm{I}} & =\sum_{k=0}^{j} E(Y \mid X=1, K=k) \cdot \frac{P(X=1, K=k)}{P(X=1)}-\sum_{k=0}^{j} E(Y \mid X=0, K=k) \cdot \frac{P(X=0, K=k)}{P(X=0)} \\
& =[76 \cdot 0.05 / 0.50+116 \cdot 0.45 / 0.50]-[85 \cdot 0.35 / 0.50+110 \cdot 0.15 / 0.50] \\
& =112-92.5 \\
& =19.5
\end{aligned}
$$

For the ANOVA type II main effect we obtain:

$$
\begin{aligned}
M E_{10}^{\mathrm{II}} & =\sum_{k=0}^{j} Q^{i f}(Y \mid X=1, K=k) \cdot \frac{1}{1+1}-\sum_{k=0}^{j} Q^{i f}(Y \mid X=0, K=k) \cdot \frac{1}{1+1} \\
& =[85.45 \cdot 0.5+114.95 \cdot 0.5]-[83.65 \cdot 0.5+113.15 \cdot 0.5] \\
& =100.2-98.4 \\
& =1.8
\end{aligned}
$$

As mentioned above, there is an alternative computation of type II main effects for $2 \times 2$ designs based on weighted cell means $E(Y \mid X=x, K=k)$ instead of $Q^{i f}(Y \mid X=x, K=k)$ (Brandt, 1933 , p. 169; Maxwell et al., 2018, p. 365; Yates, 1934, p. 62). It reveals identical results (see Appendix A at https://osf.io/vjda8).

The ANOVA type III main effect is:

$$
\begin{aligned}
M E_{10}^{\mathrm{III}} & =\sum_{k=0}^{j} E(Y \mid X=1, K=k) \cdot \frac{1}{1+1}-\sum_{k=0}^{j} E(Y \mid X=0, K=k) \cdot \frac{1}{1+1} \\
& =[76 \cdot 0.5+116 \cdot 0.5]-[85 \cdot 0.5+110 \cdot 0.5] \\
& =96-97.5 \\
& =-1.5
\end{aligned}
$$


And finally, for the average treatment effect we obtain:

$$
\begin{aligned}
A T E_{10} & =\sum_{k=0}^{j} E(Y \mid X=1, K=k) \cdot P(K=k)-\sum_{k=0}^{j} E(Y \mid X=0, K=k) \cdot P(K=k) \\
& =[76 \cdot 0.40+116 \cdot 0.60]-[85 \cdot 0.40+110 \cdot 0.60] \\
& =100-100 \\
& =0
\end{aligned}
$$

The main effects for our unbalanced $2 \times 2$ example differ strongly in size and direction. They vary from -1.5 (type III) to 19.5 (type I) while the ATE shows no treatment effect at all. Consequently, the results lead to considerably different interpretations regarding the effectiveness of the treatment. In our example, the main effects do not allow for a causal interpretation as average or overall effect. The next section provides a graphical illustration of the example and the different effects.

\section{Graphical Illustration}

Figure 1 provides a graphical illustration of our minimal $2 \times 2$ example including the ANOVA main effects and the ATE. The horizontal axis depicts the covariate $(K=0$ for women, $K=1$ for men). Colors of the circles and the regression lines indicate treatment groups $(X=1$ in dark gray, $X=0$ in light gray). The circles correspond to the numbers given in Table 2, Their centers represent the population means $E(Y \mid X=x, K=k)$ and their areas resemble the cell probabilities $P(X=x, K=k)$. Figure 1 shows some characteristics of the minimal example above: Men tend to be in the treatment group $(X=1)$, whereas women tend to be in the control group $(X=0)$. However, in sum, there are more men than women in the example as the total area of the circles on the right is larger than the total area of the circles on the left. Finally, the regression lines $E(Y \mid X=1, K)$ and $E(Y \mid X=0, K)$ are not parallel and do even cross. Thus, there is an interaction. The dashed lines represent the linear quasi-regression $Q^{i f}(Y \mid X, K)$ that ignores interactions. Therefore, these two lines are parallel and a least-squares criterion can be used for 


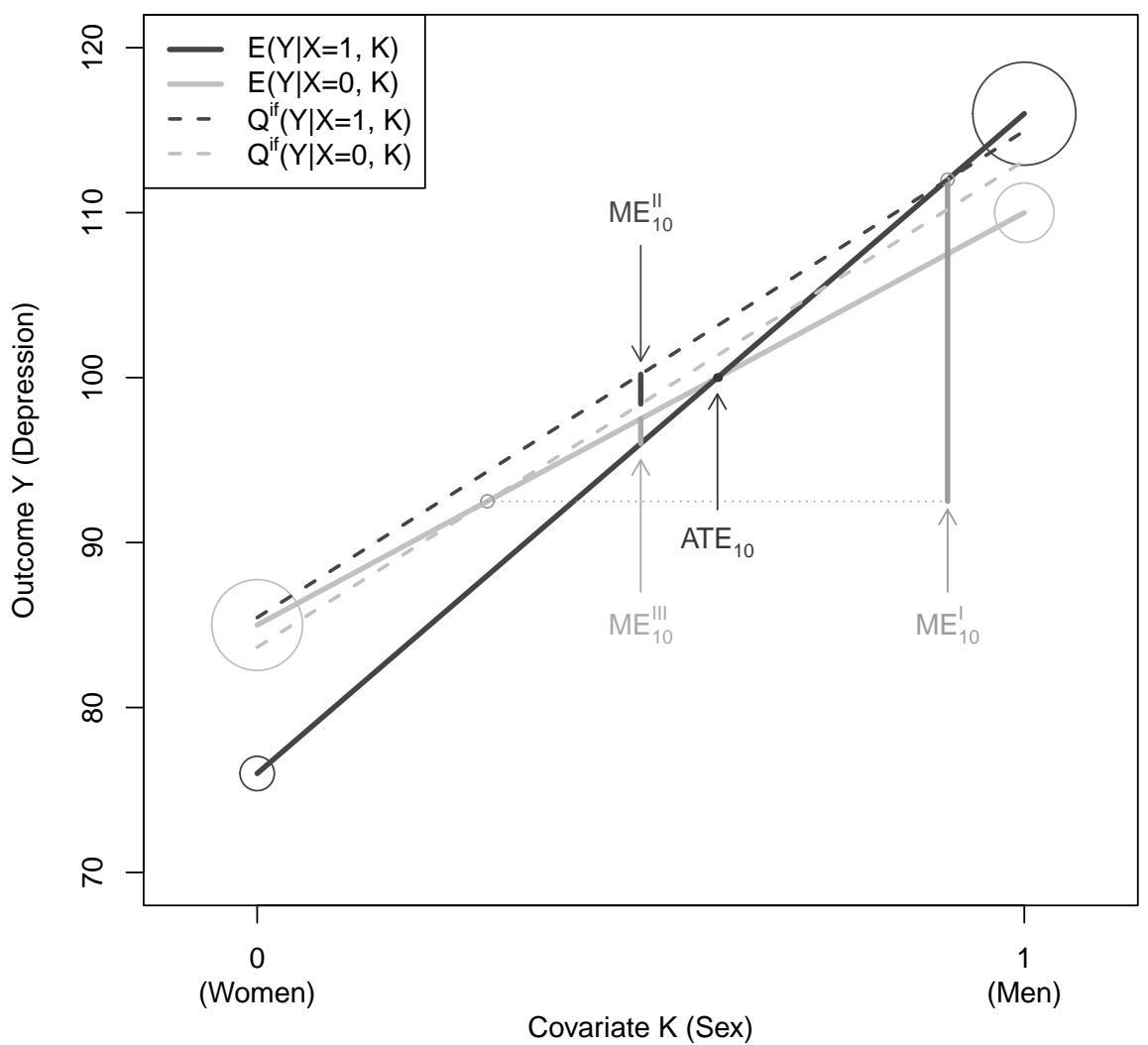

\section{Figure 1}

ANOVA type I, II, and III main effects $\left(M E_{10}\right)$ of $X$ and average treatment effect $(A T E)$ for the minimal $2 \times 2$ example. Ascending lines represent regressions (with interaction) and quasi-regressions (without interaction) for the two treatment groups ( $X=0$ : control, $X=1$ : cognitive behavioral therapy). Sizes of circles indicate group sizes. Lengths of vertical lines correspond to effect sizes of $M E_{10}$ and $A T E$.

fitting them. Population means $E(Y \mid X=x, K=k)$ and probabilities $P(X=x, K=k)$ matter for the location of these lines: The lines tend to be closer to the population means that occur with higher probability.

ANOVA main effects and ATE correspond to differences between specific points on these lines. Note that ANOVA type I and III main effects and the ATE are based on values of the genuine regression while ANOVA type II is based on values of the quasi-regression. For the ANOVA type I main effect, population means are weighted taking the distribution of the covariate in each treatment group into account: As there are more men in CBT group $X=1$, the point 
representing the weighted mean in the treatment group is on the right at $K=0.9$. Further, as there are more women in the control group $X=0$, the corresponding point for the control group is on the left at $K=0.3$. The type I main effect thus corresponds to the difference between $E(Y \mid X=1, K=0.9)$ and $E(Y \mid X=0, K=0.3)$. The dotted horizontal line facilitates the comparison of these two values. The ANOVA type II main effect simply corresponds to the difference between the two lines representing the linear quasi-regression. We draw this difference at $K=0.5$ according to the computations in the minimal example above, where equal weights are used for weighting means within each treatment group. However, as the two quasi-regression lines are parallel, the difference is identical at every point on the horizontal axis. ANOVA type III uses equal weights for all cells and relies on the genuine regressions. Its main effect is represented by the difference between the regression lines at the midpoint of the horizontal axis, i.e., at $K=0.5$. At this point, the regression line of the CBT group is lower than the regression line of the control group, resulting in a slightly negative ANOVA type III main effect. Finally, the ATE takes the marginal distribution of the covariate across treatment groups into account. It weights the population means using the marginal distribution for both treatment groups. For our example $E(K)=0.6$, thus the ATE is represented by the difference of the regression lines at $K=0.6$. At this value both regression lines intersect and the ATE is 0 .

The minimal example and its graphical illustration show that ANOVA main effects and ATE may differ strongly in size and direction. The next section provides theoretical derivations, in which designs and under which conditions ANOVA main effects correspond to the ATE. These derivations are again general and not restricted to the $2 \times 2$ design.

\section{When are ANOVA Main Effects Equal to Average Treatment Effects?}

For understanding the conditions under which ANOVA main effects are equal to average treatment effects, we start by describing three different research designs and their mathematical properties as well as further properties of the data. Based on these properties and the definitions introduced above, we show under which circumstances different ANOVA main effects equal the 
ATE.

\section{Properties of Research Designs and Models Considered}

There are many different research designs and various ways of systematizing them (e.g., Cooper et al., 2012; Kirk, 2013; Maxwell et al., 2018; Shadish et al., 2002). For this article, we focus on research designs that are multifactorial and focus on between-subject effects. Further, we assume the sample to be representative and the group sizes to be stochastic. Additionally, the cell sizes may represent balanced, proportional, or nonorthogonal designs. For each design, models with or without interactions may apply.

As mentioned before, multifactorial between-subject designs are widespread in social sciences (e.g., Keselman et al., 1998) and allow for evaluating treatment effects for two or more groups under consideration of one or more categorical covariate(s) (e.g., Maxwell et al., 2018). By using representative sampling and stochastic group sizes, the covariate distribution can be assumed to mimic the distribution of these variables in the population (e.g., Shadish et al., 2002). Deviations may only occur due to chance. This is crucial for the computation of average treatment effects and their interpretations. Note that nonrepresentative and/or fixed group sizes may also be used for correct computations of ATEs. These cases will be addressed and briefly explained in the discussion. Additionally, the patterns of cell sizes are important for understanding the equivalence of ANOVA main effects and ATE as the different designs (balanced, proportional, nonorthogonal) entail different properties regarding the (in)dependence of factors and the corresponding cell probabilities. For each design, models with or without interactions may apply.

Balanced Designs: Balanced designs originate from experimental research and show equal cell frequencies in each combination of values of $X$ and $K$. In terms of probabilities they are characterized by equal cell probabilities in each combination of values of $X$ and $K$. By definition, the factors in balanced designs are orthogonal, since the cell probabilities can always be obtained by multiplying the corresponding marginal probabilities. Consequently, such designs have the following properties regarding the stochastic independence of factors (Equations 1 to 3) and 
regarding the uniform distribution of each factor (Equations 4 and 5):

$$
\begin{gathered}
P(X=x, K=k)=P(X=x) \cdot P(K=k) \\
P(X=x)=P(X=x \mid K=k) \\
P(K=k)=P(K=k \mid X=x) \\
P(X=x)=\frac{1}{1+p} \\
P(K=k)=\frac{1}{1+j}
\end{gathered}
$$

Proportional Designs: The second research design that we consider is a so called proportional design. This is an unbalanced design with unequal cell frequencies, where the cell probabilities are proportional across rows and columns (e.g., Driscoll \& Borror, 2000). In these designs, factors $X$ and $K$ are stochastically independent, i.e., cell probabilities can be obtained by multiplying the corresponding marginal probabilities. Consequently, in proportional designs Equations 1, 2, and 3 hold. For the purpose of this article, it does not matter whether Equation 4 holds or not, because we focus on the effect of $X$ and for this, the marginal distribution of $K$ is relevant. Regarding the marginal distribution of $K$ we assume that Equation 5 does not hold in order to distinguish the proportional design from the balanced one.

Nonorthogonal Designs: The last design that we consider is an unbalanced nonproportional design with unequal cell frequencies, also known as nonorthogonal design. In this design, the cell probabilities are unequal and the factors are not independent. Consequently, none of the properties concerning stochastic independence (Equations 11 to 3) apply to these designs. In these designs, Equations 4 and 5 may or may not hold.

Models With(out) Interaction: In models without interaction between $X$ and $K$, the genuine regression $E(Y \mid X, K)$ is equal to the interaction free quasi-regression $Q^{i f}(Y \mid X, K)$. Using Wilkinson and Rogers notation, both can then be represented by $Y \sim 1+X+K$. Consequently, 
Table 3

When are ANOVA main effects equal to average treatment effects?

\begin{tabular}{ccccccc}
\hline & \multicolumn{2}{c}{ Balanced } & \multicolumn{2}{c}{ Proportional } & \multicolumn{2}{c}{ Nonorthogonal } \\
ANOVA & No Interaction & Interaction & No Interaction & Interaction & No Interaction & Interaction \\
\hline Type I & $\checkmark$ & $\checkmark$ & $\checkmark$ & $\checkmark$ & X & X \\
Type II & $\checkmark$ & $\checkmark$ & $\checkmark$ & $\checkmark$ & $\checkmark$ & X \\
Type III & $\checkmark$ & $\checkmark$ & $\checkmark$ & $X$ & $\checkmark$ & X \\
\hline
\end{tabular}

Note. For all conditions, conditional regressive dependence of $Y$ and $X$ is assumed. " $\checkmark$ " indicates equivalence to average treatment effects, " $X$ " indicates non-equivalence.

also the predicted values of these regressions are equal as well in models without interaction:

$$
E(Y \mid X=x, K=k)=Q^{i f}(Y \mid X=x, K=k) .
$$

In models with interactions this equation does not hold.

\section{Equivalence of ANOVA Main Effects and Average Treatment Effects}

For examining the equivalence of ANOVA main effects and average treatment effects we assume that both $X$ and $K$ have a conditional effect on $Y$. That means, there are effects of $X$ on $Y$ given $K=k$ as well as of $K$ on $Y$ given $X=x$. We further assume that $K$ is the only relevant covariate, i.e., that there are no other confounding variables. Table 3 provides an overview under which conditions average treatment effects and ANOVA main effects coincide.

ANOVA Type I. For the equivalence of average treatment effects and ANOVA type I main effects the following equality must hold:

$$
\begin{aligned}
A T E_{x x^{\prime}} & =\sum_{k=0}^{j} E(Y \mid X=x, K=k) \cdot P(K=k) \quad-\sum_{k=0}^{j} E\left(Y \mid X=x^{\prime}, K=k\right) \cdot P(K=k) \\
& =\sum_{k=0}^{j} E(Y \mid X=x, K=k) \cdot P(K=k \mid X=x)-\sum_{k=0}^{j} E\left(Y \mid X=x^{\prime}, K=k\right) \cdot P\left(K=k \mid X=x^{\prime}\right)
\end{aligned}
$$




$$
=M E_{x x^{\prime}}^{\mathrm{I}}
$$

Consequently, the two effects are equal if $P(K=k)=P(K=k \mid X=x)=P\left(K=k \mid X=x^{\prime}\right)$. This applies to balanced and proportional designs (cf. Equation 3). It applies to both, models with and without interactions, because the computations of $A T E_{x x^{\prime}}$ and $M E_{x x^{\prime}}^{\mathrm{I}}$ are both based on the same conditional expectations. In nonorthogonal designs, Equation 3 does not hold, and therefore, ATE and ANOVA type I main effects are different.

ANOVA Type II. For the equivalence of average treatment effects and ANOVA type II main effects the following equality must hold:

$$
\begin{aligned}
A T E_{x x^{\prime}} & =\sum_{k=0}^{j} E(Y \mid X=x, K=k) \cdot P(K=k)-\sum_{k=0}^{j} E\left(Y \mid X=x^{\prime}, K=k\right) \cdot P(K=k) \\
& =\sum_{k=0}^{j} Q^{i f}(Y \mid X=x, K=k) \cdot \frac{1}{j+1}-\sum_{k=0}^{j} Q^{i f}\left(Y \mid X=x^{\prime}, K=k\right) \cdot \frac{1}{j+1} \\
& =M E_{x x^{\prime}}^{\mathrm{II}}
\end{aligned}
$$

There are three conditions where this equality holds. First, obviously, this equation is true if $P(K=k)=\frac{1}{1+j}$ and $E(Y \mid X=x, K=k)=Q^{i f}(Y \mid X=x, K=k)$, which applies to balanced designs without interaction (cf. Equations 5 and 6). This is fairly restrictive but there are weaker conditions. In fact, either the absence of an interaction (see second condition) or a balanced design on its own (see third condition) are sufficient for the main effect of ANOVA type II being equal to the ATE as we will show next. Second, to see why the absence of interaction is sufficient, consider that $P(K=k)$ and $\frac{1}{j+1}$ can be factored out in the equality above. If the remaining differences are equal, i.e.,

$$
E(Y \mid X=x, K=k)-E\left(Y \mid X=x^{\prime}, K=k\right)=Q^{i f}(Y \mid X=x, K=k)-Q^{i f}\left(Y \mid X=x^{\prime}, K=k\right)
$$

for all $K=k$, the sums of the differences add up to the same value, because in this case, the weighting factor is irrelevant. Consequently, $A T E_{x x^{\prime}}=M E_{x x^{\prime}}^{\mathrm{II}}$ is true for models without 
interactions in all designs. Third, to see why independence of $X$ and $K$ is sufficient for the equivalence of ANOVA main effect type II and ATE (even in the presence of interactions), consider the definitions based on model comparisons introduced above and repeated here:

$$
\begin{array}{llll}
M E^{\mathrm{II}}: & Y \sim 1+X^{\mathrm{eff}}+K^{\mathrm{eff}} & \text { vs. } & Y \sim 1+K^{\mathrm{eff}} \\
\text { ATE }: & Y \sim 1+X^{\mathrm{dum}}+K^{\mathrm{cen}}+X^{\mathrm{dum}} \cdot K^{\mathrm{cen}} & \text { vs. } & Y \sim 1+K^{\mathrm{cen}}+X^{\mathrm{dum}} \cdot K^{\mathrm{cen}}
\end{array}
$$

Note that the two definitions differ with regard to the interaction term. So both model comparisons yield the same result for the effect of $X$ if the interaction term $X^{\mathrm{dum}} \cdot K^{\mathrm{cen}}$ is independent of $X$. This is true for designs with randomized and mean centered factors (e.g., Cohen et al., 2003, chapter 7). In the terminology used in this article, this applies to all balanced and proportional designs, no matter whether there are interactions or not.

ANOVA Type III. For the equivalence of ANOVA type III main effects and average treatment effects the following equality must hold:

$$
\begin{aligned}
A T E_{x x^{\prime}} & =\sum_{k=0}^{j} E(Y \mid X=x, K=k) \cdot P(K=k)-\sum_{k=0}^{j} E\left(Y \mid X=x^{\prime}, K=k\right) \cdot P(K=k) \\
& =\sum_{k=0}^{j} E(Y \mid X=x, K=k) \cdot \frac{1}{j+1} \quad-\sum_{k=0}^{j} E\left(Y \mid X=x^{\prime}, K=k\right) \cdot \frac{1}{j+1} \\
& =M E_{x x^{\prime}}^{\mathrm{III}}
\end{aligned}
$$

This equality holds in two cases: First, it holds if the marginal probability of $K$ is equal to the type III weights, i.e., $P(K=k)=\frac{1}{1+j}$, which applies to balanced designs for models with and without interactions (cf. Equation 5). Second, $A T E_{x x^{\prime}}=M E_{x x^{\prime}}^{\mathrm{III}}$ if the factored out differences $E(Y \mid X=x, K=k)-E\left(Y \mid X=x^{\prime}, K=k\right)$ are the same for all $K=k$, because in this case, the weighting factor is irrelevant. The latter is true for models without interactions in all designs.

\section{Simulation Study}

To complement our theoretical derivations on the equality of ANOVA main effects and average treatment effects (cf. Table 3), we conducted a simulation study in order to examine the 
severity of bias under different conditions and the practical relevance of our findings.

\section{Data Generation}

In this simulation study, we aim at confirming and extending our theoretical results. This includes to examine the severity of bias for possible problematic conditions. We used a simple $2 \times 2$-factorial design that mimics our previous example with two treatment conditions $(X=1$ as treatment group, $X=0$ as control group) and two covariate values $(K=0$ and $K=1)$. As a possible causal interpretation of main effects is of major interest, the ATE was set to equal zero $(A T E=0)$ and all conditions were simulated under this null hypothesis. Results of supplementary power analyses with $A T E=5$ are provided at https://osf.io/vjda8 in Appendix C Taking previous theoretical results as a basis, we varied two factors in our simulation study, first, designs with levels (A) balanced designs, (B) unbalanced proportional designs, and (C) nonorthogonal designs, and second, size of interaction with levels (1) no interaction, (2) medium interaction, and (3) large interaction. Based on these variations, population means and corresponding probabilities were deduced for all conditions. Table 4 shows an overview of all combinations. Differences in probabilities represent different research designs, differences in population means of the cells represent different sizes of interaction taking $A T E=0$ into account. Total sample size of each sample was set to $N=120$ with fixed group sizes corresponding to the theoretical probabilities. To each population mean of Table 4 , a random term was added with $\varepsilon \sim \mathcal{N}(0,15)$. For each simulation condition $R=10,000$ data sets were generated. The simulation study was conducted using R ( $\mathrm{R}$ Core Team, 2020). The package SimDesign was used for data generation, analyses, and summary of results (Sigal \& Chalmers, 2016).

\section{Analysis}

For every dataset four different analyses were conducted: ANOVA type I, ANOVA type II, ANOVA type III, and ATE computation. For the computation of ANOVA type I with $X$ entered first, the R default setting (anova() function of the stats package) was used (R Core Team, 


\section{Table 4}

Simulation: Population means $E(Y \mid X=x, K=k)$ and corresponding probabilities $P(X=x, K=k)$ in italics for all treatment $\times$ covariate conditions of different research designs and sizes of interaction

\begin{tabular}{|c|c|c|c|c|c|c|}
\hline \multirow{3}{*}{$\begin{array}{l}\text { Design } \\
\text { (A) Balanced }\end{array}$} & \multirow{4}{*}{$\frac{\text { Interaction }}{\text { (1) No }}$} & \multirow{4}{*}{$\begin{array}{l}\text { Treatment } \\
X=0 \\
X=1\end{array}$} & \multicolumn{4}{|c|}{ Covariate } \\
\hline & & & \multicolumn{2}{|c|}{$K=0$} & \multicolumn{2}{|c|}{$K=1$} \\
\hline & & & 80 & 0.25 & 110 & 0.25 \\
\hline & & & 80 & 0.25 & 110 & 0.25 \\
\hline & (2) Medium & $X=0$ & 80 & 0.25 & 110 & 0.25 \\
\hline & & $X=1$ & 75.5 & 0.25 & 114.5 & 0.25 \\
\hline & (3) Large & $X=0$ & 80 & 0.25 & 110 & 0.25 \\
\hline & & $X=1$ & 71 & 0.25 & 119 & 0.25 \\
\hline \multirow[t]{6}{*}{ (B) Proportional } & (1) $\mathrm{No}$ & $X=0$ & 80 & 0.17 & 110 & 0.33 \\
\hline & & $X=1$ & 80 & 0.17 & 110 & 0.33 \\
\hline & (2) Medium & $X=0$ & 80 & 0.17 & 110 & 0.33 \\
\hline & & $X=1$ & 74 & 0.17 & 113 & 0.33 \\
\hline & (3) Large & $X=0$ & 80 & 0.17 & 110 & 0.33 \\
\hline & & $X=1$ & 68 & 0.17 & 116 & 0.33 \\
\hline \multirow[t]{6}{*}{ (C) Nonorthogonal } & (1) No & $X=0$ & 80 & 0.29 & 110 & 0.21 \\
\hline & & $X=1$ & 80 & 0.04 & 110 & 0.46 \\
\hline & (2) Medium & $X=0$ & 80 & 0.29 & 110 & 0.21 \\
\hline & & $X=1$ & 74 & 0.04 & 113 & 0.46 \\
\hline & (3) Large & $X=0$ & 80 & 0.29 & 110 & 0.21 \\
\hline & & $X=1$ & 68 & 0.04 & 116 & 0.46 \\
\hline
\end{tabular}

Note. Marginal probabilities of treatment variable $X$ are $P(X=x)=0.5$ in all conditions. Marginal probabilities of the covariate $K$ are $P(K=k)=0.5$ in all balanced designs and $P(K=0)=0.33$ and $P(K=1)=0.67$ in all proportional and nonorthogonal designs respectively.

2020). ANOVA type II and III were implemented using the car package (version 2.1-4, Fox \& Weisberg, 2010). For type III analyses, orthogonal contrasts were used.

For the ATE computation, the package EffectLiteR was used (Mayer et al., 2016). The EffectLiteR model was specified in such a way that it is comparable to the other approaches, i.e., assuming fixed cell sizes and homoscedastic error variances. Using the option method="lm", the package provides direct estimations of the ATE as well as corresponding $F$ - and $p$-values. The 
results of ATE estimation via EffectLiteR corresponded exactly to the ATE estimations of Robin's g-formula without time-varying variables yielded by the package gfoRmula (McGrath et al., 2020). For brevity, we restrict the presentation of results to EffectLiteR, as its output allows for direct and easy comparisons to results of ANOVA models.

We considered bias as outcome criterion. That is, the deviation of the estimated effect from the generated ATE of zero. In order to obtain summary measures for the different simulation conditions, the average bias of all replications within one condition (Bias) and its standard deviation are studied. Furthermore, the empirical detection rate (EDR) for Type I errors of the main effect of $X$ or the ATE were analyzed, using the EDR() function (Sigal \& Chalmers, 2016). For every simulation condition, the EDR gives the proportion of main effects or ATEs that was significantly different from zero. The critical $\alpha$ level was set to .05 , so the EDR should vary around that value in order to meet the Type I error assumed. Corresponding results are presented below. Additionally, the root mean square error (RMSE) was studied (cf. Appendix Cat https://osf.io/vjda8 for results).

\section{Results}

Biases for every simulation condition are presented first. Afterwards, results for empirical detection rates are described. The results are structured regarding (A) balanced, (B) proportional, and (C) nonorthogonal research designs. Within these paragraphs we review results for different analysis approaches and sizes of interactions. If necessary, superscripts indicate ANOVA type I, II, and III main effects, and average treatment effects (ATE).

For balanced designs (A) all analysis procedures revealed the same results. This finding corresponds to well known equivalences in these procedures. Thus, results are merely graphically displayed. Exact numbers can be found in Appendix Cat https://osf.io/vjda8.

\section{Bias}

A graphical overview of results for all simulation conditions showing the bias (Bias) of ATE estimation and its standard deviation for all analysis approaches is provided in Figure 2. 

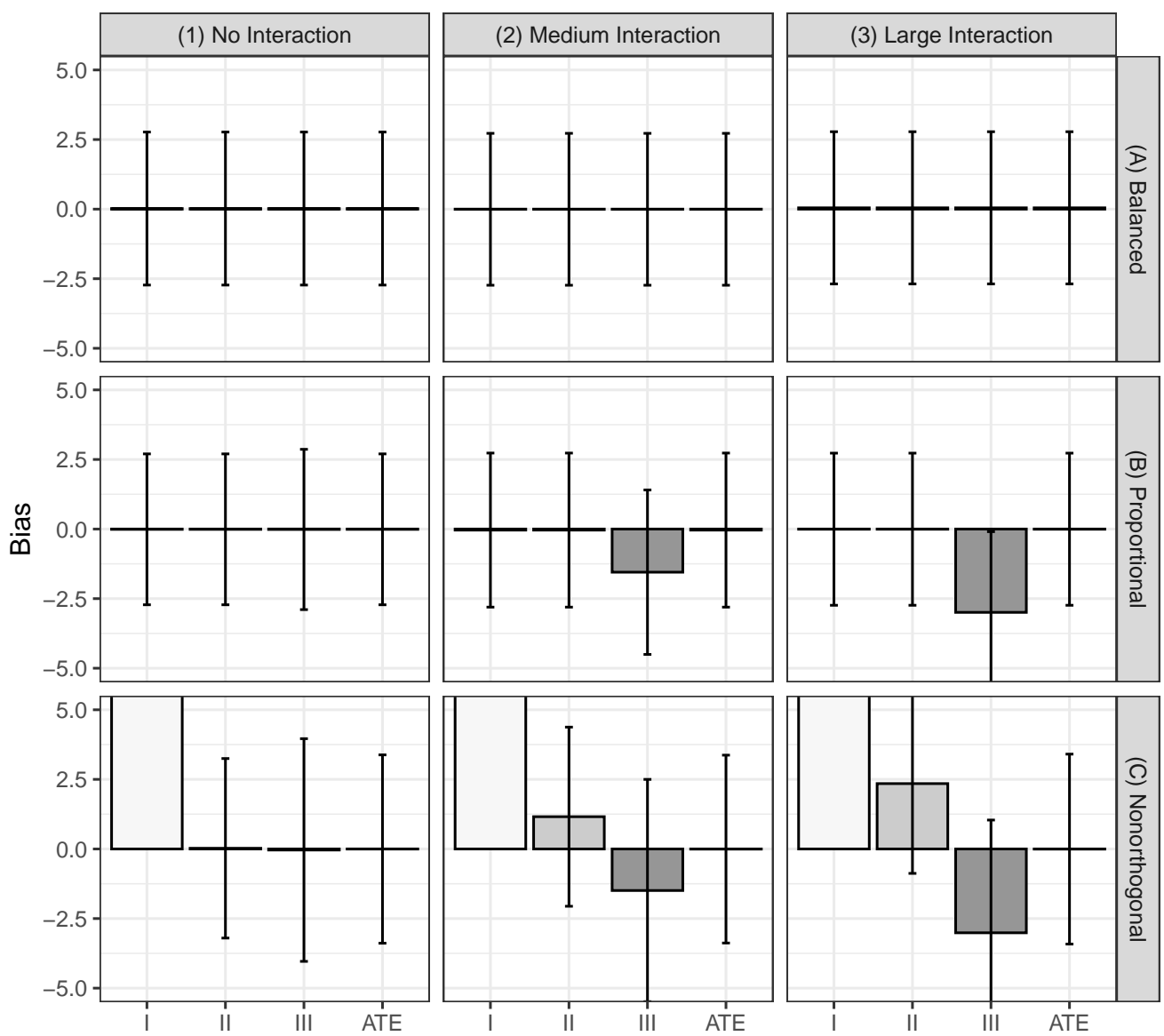

Approach

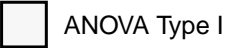

ANOVA Type II

ANOVA Type III ATE

Figure 2

Biases of the estimation of ANOVA main effects of X (type I, II, III) and average treatment effects (ATE) with corresponding standard deviations for different research designs $(A, B, C)$ and sizes of interactions $(1,2,3)$

(B) Proportional Research Design. Similar results as for balanced designs were found for proportional research designs. Except for ANOVA type III, all analysis approaches led to biases around zero and can, thus, be expected to be unbiased. The standard deviations varied slightly around $S D=2.7$ for different sizes of interaction. For ANOVA type III, the bias was only close to zero $(S D=2.9)$ if no interaction was present. For a medium interaction, the bias was Bias $^{\mathrm{III}}=-1.5(S D=2.9)$, while for a large interaction it increased towards Bias $^{\mathrm{III}}=-3$ $(S D=2.9)$.

(C) Nonorthogonal Research Design. The most obvious deviations from the underlying null hypothesis of $A T E=0$ were found for nonorthogonal research designs. ANOVA 
type I failed completely to meet an ATE of zero. For all sizes of interaction, the estimated treatment effect was clearly overestimated. If no interaction was present, the bias was Bias $^{\mathrm{I}}=15.0(S D=2.7)$ on average. For medium interaction, the bias was Bias $^{\mathrm{I}}=17.3$ $(S D=2.8)$, while for large interaction it increased towards Bias $^{\mathrm{I}}=19.5(S D=2.7)$.

If no interaction was present, all remaining approaches yielded a bias close to zero on average. Compared to the other research designs, the corresponding standard deviations increased to $S D=3.2$ for ANOVA type II, to $S D=4.0$ for ANOVA type III, and to $S D=3.4$ for ATE computation. In presence of interactions, ANOVA type II and III led to systematically biased estimates of ATE. For medium interactions, the bias for ANOVA type II was Bias $^{\mathrm{II}}=1.2$ $(S D=3.3)$ and for ANOVA type III Bias ${ }^{\mathrm{III}}=-1.4(S D=4.0)$. For large interactions, the bias ${\text { increased to } \text { Bias }^{\mathrm{II}}=2.4(S D=3.2) \text { for type II and to Bias }}^{\mathrm{III}}=-3.0(S D=4.0)$ for type III. Results for ATE estimation via EffectLiteR were very similar with Bias $^{\mathrm{ATE}}=0.1(S D=3.4)$ for models with medium interaction and Bias $^{\mathrm{ATE}}=0.0(S D=3.4)$ for models with large interaction. That means, only this approach led to an unbiased estimate of ATE in nonorthogonal designs with interaction.

\section{Empirical Detection Rate of Type I Error}

A graphical overview of results for all simulation conditions showing the empirical detection rate $(\mathrm{EDR})$ of Type I error under the null hypothesis $A T E=0$ for all analysis approaches is provided in Figure 3 .

(B) Proportional Research Design. Similar results as for balanced designs were found for proportional research designs, except for ANOVA type III. Results for EDR were .050 for ANOVA type I, type II, and ATE computation if no interaction was present, .046 for medium interaction, and .045 for large interaction. ANOVA type III showed an EDR of .051 (no interaction), .080 (medium interaction), and .172 (large interaction). The nominal $\alpha$ level was only met in the condition without interaction. For conditions with interaction, the rejection rate of the null hypothesis $A T E=0$ was clearly above .05 . Thus, ANOVA type III resulted in a 


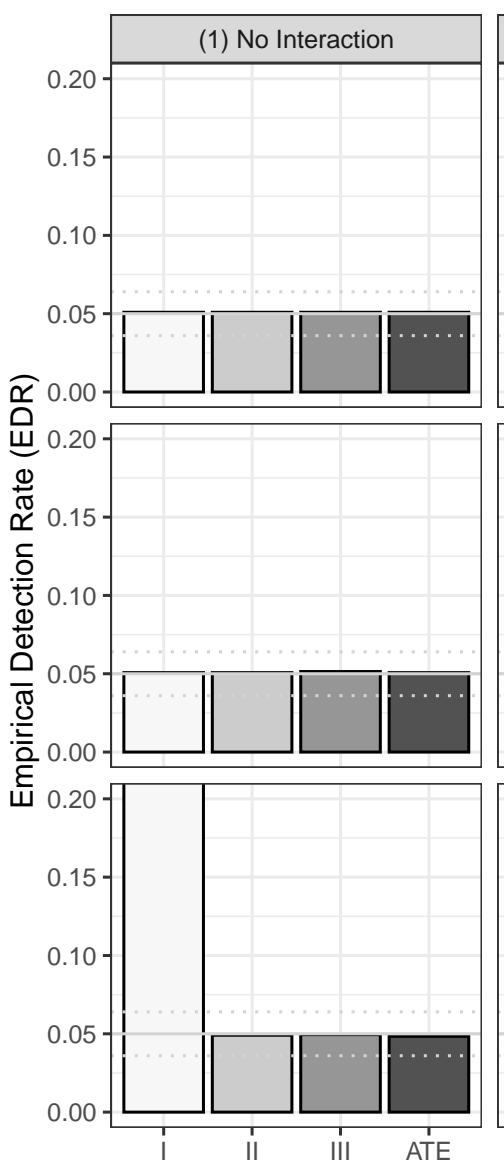

Figure 3
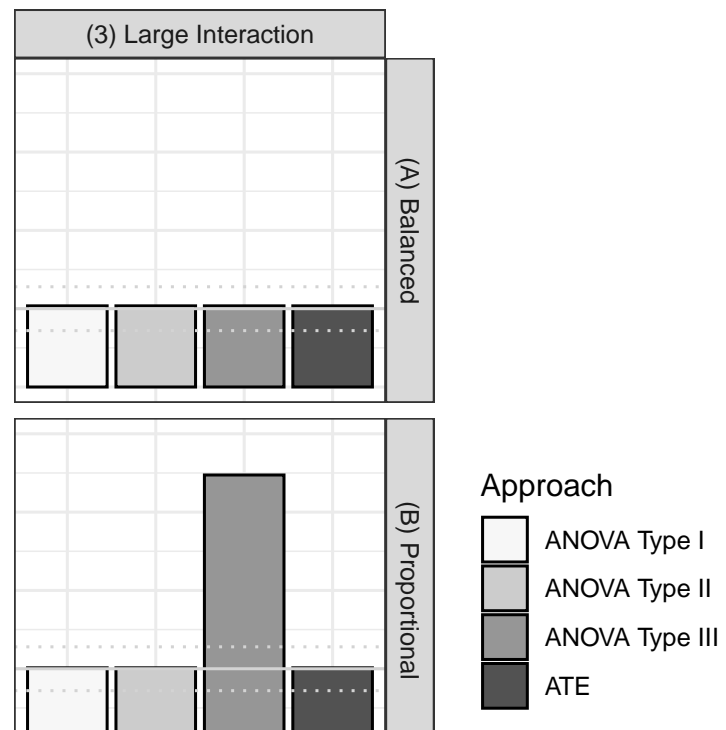

Empirical detection rate (EDR) of Type I error for different research designs $(A, B, C)$ and sizes of interactions (1, 2, 3). The gray solid line shows the theoretically assumed EDR of .05. The gray dotted lines represent the 95-\% confidence interval around .05.

increasingly liberal test for null hypotheses for conditions with higher interactions.

(C) Nonorthogonal Research Design. In nonorthogonal designs, EDRs of Type I error do considerably depend on size of interaction and the applied analysis approach. For models without interaction, results are similar to the other research designs except for ANOVA type I. Here, the effect of the treatment was (almost) always assumed to be significant different from zero $(E D R=1.0)$. The EDR of the other approaches varied from .047 for ANOVA type II, over .051 for ANOVA type III to .049 for ATE computation.

For conditions with interaction the EDR for ANOVA type I stayed at the maximum of 1.0. Rates increased for ANOVA type II and III for higher interactions. For medium interaction, we 
found an EDR of .067 for ANOVA type II and .065 for type III. For large interaction, EDRs increased further towards .113 for both, ANOVA type II and type III.

In contrast to that, ATE computation stayed stable within a 95-\% confidence interval around the $\alpha$ level of .05. It resulted in an EDR of .051 for medium interaction and .052 for large interaction.

\section{Summary of Simulation Study}

The simulation study underpins the observations from the theoretical derivations above (cf. Table 3): Although the effects estimated and tested by different ANOVA approaches may coincide with the ATE in some conditions, no ANOVA approach is adequate for testing the ATE under arbitrary conditions. All ANOVA approaches revealed the same results for balanced designs. They appropriately estimated the ATE. In unbalanced designs and/or in conditions containing an interaction, the ANOVA approaches resulted in substantial deviations from the ATE. In proportional designs with interactions, ANOVA type III showed negative biases on average and relevant deviations from the nominal $\alpha$ level of .05 . In nonorthogonal designs, all ANOVA approaches deviate from the ATE and the nominal $\alpha$ level of .05, especially for models with interactions. ANOVA type I yielded substantial deviations even for models without interactions. ATE computation provided equal results for balanced designs and appropriately estimated the ATE. Additionally, ATE computation led to satisfying ATE estimations and EDRs in all conditions including nonorthogonal designs containing an interaction. With regard to simulation under the alternative hypothesis, in all valid cases all procedures yielded the same or at least comparable sizes of power.

\section{Discussion}

\section{Summary}

In this contribution we focused on the equivalence of ANOVA main effects and average

treatment effects. Since many researchers using ANOVA may have causal interpretations in mind, 
we show under which conditions main effects and average treatment effects are the same. While there are considerably different definitions of ANOVA main effects (often known as type I, II, and III), the ATE is well defined in the causal inference literature. In this article, we introduced all effects in a consistent manner by means of effect computations and model comparisons. A minimal $2 \times 2$ nonorthogonal design example and its graphical illustration showed substantial differences between main effects obtained from ANOVA type I, type II, and type III and the average treatment effect. Afterwards, we derived theoretically, not limited to a $2 \times 2$ design anymore, under which conditions ANOVA main effects and ATE are the same. We considered balanced, proportional, and nonorthogonal research designs and models with and without interactions. Additionally, the severity of bias and the empirical detection rate were examined in a simulation study. For the respective procedures, this means:

\section{ANOVA Type I}

ANOVA type I is adequate in balanced and proportional designs, which may be achieved by randomized treatment allocation, for example. However, this approach fails in nonorthogonal designs.

\section{ANOVA Type II}

This approach is often recommended to be used only in the absence of an interaction (cf. Appelbaum \& Cramer, 1974). The derivation above and the results of the simulation study show that even in the presence of an interaction this procedure can successfully be used in balanced and proportional designs, but not in nonorthogonal designs with an additional interaction.

\section{ANOVA Type III}

ANOVA type III also requires absence of an interaction or alternatively a balanced design. As the theoretical derivations showed, the critical point about the design in this case is the uniform distribution of the covariate. A uniform distribution of the covariate might be realistic only for a minority of cases (e.g., sex if its distribution is $50 \%$ female and $50 \%$ male), but not for 
many other covariates.

\section{ATE Computation}

Of course, an approach that is designed to estimate and test the ATE should lead to satisfying results. Such an approach based on the linear model in EffectLiteR was also included in the simulation study. It provided equal results like the other approaches for balanced designs and satisfying ATE estimations and EDR in all conditions including nonorthogonal designs containing an interaction.

\section{Recommendations for Applied Researchers}

Based on these results, different approaches can be used: Although the assumption of no interaction between the treatment variable $X$ and the covariate $K$ is pretty strong, in this case ANOVA type II and III as well as the ATE computation can successfully be applied. Further, in cases with proportional designs, e.g., in randomized experiments, ANOVA type I, type II, or the ATE computation can be used. Because of their impact, it is important to think of the research design and the distribution(s) of covariate(s) carefully in advance. As the ATE computation yielded equal or better results in all conditions when compared to the other approaches, we recommend to use this approach for testing the ATE when accounting for categorical covariates. Note that if all relevant covariates are taken into account and there are no additional confounding variables, causal inferences can be drawn. Alternatively to ATE computation via EffectLiteR, this approach can be implemented by using adequate contrasts and mean centering in standard statistical software. In general, the ATE computation also allows for considering even complex designs with more treatment variables or covariates, as it can be simplified to a two-factorial design by using vectors of variables (Wüthrich-Martone, 2001, chap. 6). However, for applied researchers it may be more convenient to use EffectLiteR as it provides a graphical user interface and takes care of the correct computation for average effects even for many covariates automatically. 


\section{Practical Implications, Limitations, and Further Issues}

Throughout this article, we focused on the ATE as the effect of interest. Regarding the shortcomings of the different ANOVA approaches and their differing application recommendations, we want to emphasize that the ATE is a sensible effect and meaningful even in the presence of an interaction. We illustrate this idea by two examples from different fields: (1) Health care providers oftentimes need to rely on average effects for implementing common standards and guidelines. While it is of course desirable to deliver personalized interventions based on patient characteristics whenever possible, sometimes these characteristics are simply not available, or there is not enough time for additional assessments, or there is a limited amount of treatments or medication available, or the guidelines should be generally valid. In such cases the average effect informs about the treatment that is most effective over all patients. For example, working at an emergency department, physicians have to make decisions about the treatment for a new patient based on limited information and time. They need to decide quickly which intervention leads to the best outcome given limited pre-information. In this case, the average effect is their best guess. (2) Another example comes from educational policy where politicians need to think about the effects of changes in the standard curriculum. Politicians are probably interested in the effects of this intervention on the educational success of students and schools. Most likely, there will be students and schools where the change in curriculum has a positive effect on educational success. At the same time, there will also be students and schools where there is no effect or even a negative effect on educational success. Policy makers need to decide the best outcome for as many students as possible irrespective of some individual disadvantages. In such cases, the decision of the policy makers whether to widely implement the new curriculum or not should be based on the average effect. Another reason to consider average effects in addition to conditional effects could be a legally required equal treatment of schools or students that makes it impossible to differentiate. Our examples show that the consideration of average effects can be important even when interactions are present. Of course, the more information is taken into account the more precise one can evaluate outcomes of treatments for individuals. For 
this, conditional or interaction effects are better suited and more informative. Nevertheless, we argue that it is still worthwhile to ask research questions regarding main or average treatment effects, as there are situations where they are appropriate and meaningful.

For the ATE being a meaningful effect, however, a representative sample or knowledge of the distribution of the covariate is essential. If the distribution of the covariate in the sample does not reflect the distribution in the population of interest, all approaches may result in incorrect estimations of ATEs and the computed average treatment effects themselves do not represent average causal effects but arbitrary computed differences between means. In this case, conditional treatment effects can and should be interpreted. Alternatively, in some cases it is possible to consider the theoretically underlying distribution of the covariate in order to estimate the ATE correctly. This distribution might be found, for example, in former studies or census data. Considering this knowledge, correct weights can be used (cf. section of effect definitions using effect computations). These weights can be implemented, for example, by defining correct contrasts representing the representative sample distribution.

In designs with more than one treatment factor, the computation of average effects (as an average over the other treatment factors) might be less interesting for interpretation. From a statistical point of view its computation is still correct.

Further limitations concern the stochasticity of group sizes: As ANOVA applications are based on the assumptions of the general linear model, the group sizes are assumed to be fixed. This allows for correct point estimates of effects but ignores the possible uncertainty due to stochastic sampling. Nevertheless, research designs that return fixed instead of stochastic group sizes allow for causal interpretation of average treatment effects if the fixed distribution of covariate(s) represents the distribution of the underlying population. For example, experimental designs commonly rely on such known or planned covariate distributions and apply ANOVA with fixed factors for analyses. For this reason, many descriptions of different ANOVA types use frequencies as weights instead of probabilities. The frequencies can be used to estimate (stochastic) probabilities and lead to correct effect estimates, too. Approaches of causality 
consider this stochasticity by default and use so-called stochastic covariates or stochastic treatment variables. There are statistical approaches to deal with this, e.g., by using multigroup structural equation models or an augmenting approach (Mayer \& Thoemmes, 2019). These approaches are also implemented in EffectLiteR. By default, this R package uses a multigroup structural equation model where the regressions of the dependent variable on the continuous covariates are estimated in each group formed by combinations of the treatment and categorical covariates. So, it does not use coding of categorical predictors but a multigroup approach. Based on the parameters of the multigroup model, the average and conditional effects are computed subsequently using the multivariate delta method and the formulas provided in this paper (for the general formulas including continuous covariates, see Mayer et al., 2016). Recently, an ordinary least squares option was implemented, too. This can be used for cases with manifest variables only and fixed group sizes (see Mayer \& Thoemmes, 2019). The ordinary least squares version uses dummy coding for the implementation but the effects of interest are also computed subsequently as a function of regression coefficients and the group weights according to the formulas presented in this paper. EffectLiteR provides much more features like estimation and testing conditional effects, the use of latent variables, FIML to handle missing data, including additional continuous and categorical covariates (an example with many covariates can be found at https://osf.io/vjda8) or accounting for heteroscedastic error variances.

\section{Conclusion}

Although ANOVA approaches can be used to estimate and test the ATE in some designs and under specific conditions, they may not be adequate in other cases. There are alternative approaches that are designed to test the ATE and that can be applied in research designs commonly analyzed by ANOVA approaches. We recommend to use these alternative approaches if the effect of interest is the ATE. If additional requirements such as no additional confounding covariates hold, the results can be interpreted in a causal manner many researchers may have in mind when applying ANOVA. 


\section{Open Practices Statement}

The R code and supplementary material are available at https://osf.io/vjda8

\section{Declaration of Interest Statement}

No authors has any financial or other conflicts of interest in relation to this work. 


\section{References}

Appelbaum, M. I., \& Cramer, E. M. (1974). Some problems in the nonorthogonal analysis of variance. Psychological Bulletin, 81(6), 335-343. https://doi.org/10.1037/h0036315

Bangert, A. W., \& Baumberger, J. P. (2005). Research and statistical techniques used in the journal of counseling \& development: 1990-2001. Journal of Counseling $\mathcal{E}$ Development, 83(4), 480-487. https://doi.org/10.1002/j.1556-6678.2005.tb00369.x

Brandt, A. E. (1933). The analysis of variance in a $2 \times s$ table with disproportionate frequencies. Journal of American Statistical Association, 28(182), 164-173. https://doi.org/10.2307/2278030

Burdick, R. K. (1983). Statement of hypotheses in the analysis of variance. Journal of Marketing Research, 20(3), 320-324. https://doi.org/10.2307/3151836

Chambers, J. M., \& Hastie, T. J. (Eds.). (1991). Statistical models in S. Chapman \& Hall.

Cohen, J., Cohen, P., West, S. G., \& Aiken, L. S. (2003). Applied multiple regression/correlation analysis for the behavioral sciences. Lawrence Erlbaum.

Cooper, H., Camic, P. M., Long, D. L., Panter, A. T., Rindskopf, D., \& Sher, K. J. (Eds.). (2012). APA handbook of research methods in psychology, Vol 2: Research designs: Quantitative, qualitative, neuropsychological, and biological. American Psychological Association. https://doi.org/10.1037/13620-000

Driscoll, M. F., \& Borror, C. M. (2000). Sums of squares and expected mean squares in SAS. Quality and Reliability Engineering International, 16(5), 423-433. https://doi.org/10.1002/1099-1638(200009/10)16:5<423::aid-qre351>3.0.co;2-w

Elmore, P. B., \& Woehlke, P. L. (1998, April 13-17). Twenty years of research methods employed in "American Educational Research Journal", "Educational Researcher", and "Review of Educational Research" [Paper presentation]. Annual Meeting of the American Educational Research Association, San Diego, CA, United States. 
Elston, R. C., \& Bush, N. (1964). The hypotheses that can be tested when there are interactions in an analysis of variance model. Biometrics, 20(4), 681-698. https://doi.org/10.2307/2528122

Fisher, R. A. (1925/1973). Statistical methods for research workers (14th ed.). Hafner.

Fox, J. (2016). Applied regression analysis and generalized linear models. Sage.

Fox, J., \& Weisberg, S. (2010). An R companion to applied regression (2nd ed.). Sage.

Games, P. A. (1973). Type IV errors revisited. Psychological Bulletin, 80(4), 304-307. https://doi.org/10.1037/h0034832

Hector, A., von Felten, S., \& Schmid, B. (2010). Analysis of variance with unbalanced data: An update for ecology \& evolution. Journal of Animal Ecology, 79(2), 308-316. https://doi.org/10.1111/j.1365-2656.2009.01634.x

Hernán, M. A., \& Robins, J. M. (2020). Causal inference: What if. Chapman \& Hall/CRC. https://www.hsph.harvard.edu/miguel-hernan/causal-inference-book/

Herr, D. G., \& Gaebelein, J. W. (1978). Nonorthogonal two-way analysis of variance. Psychological Bulletin, 85(1), 207-216. https://doi.org/10.1037/0033-2909.85.1.207

Howell, D. C. (2013). Statistical methods for psychology (8th ed.). Wadsworth Cengage Learning. Imbens, G. W., \& Rubin, D. B. (2015). Causal inference for statistics, social, and biomedical sciences: An introduction. Cambridge University Press.

Keselman, H. J., Huberty, C. J., Lix, L. M., Olejnik, S., Cribbie, R. A., Donahue, B., Kowalchuk, R. K., Lowman, L. L., Petoskey, M. D., Keselman, J. C., \& Levin, J. R. (1998). Statistical practices of educational researchers: An analysis of their ANOVA, MANOVA, and ANCOVA analyses. Review of Educational Research, 68(3), 350-386. https://doi.org/10.3102/00346543068003350

Kirk, R. E. (2013). Experimental design: Procedures for the behavioral sciences. Sage. Langsrud, Ø. (2003). ANOVA for unbalanced data: Use Type II instead of Type III sums of squares. Statistics and Computing, 13(2), 163-167. https://doi.org/10.1023/a:1023260610025 
Lindstromberg, S. (2016). Inferential statistics in language teaching research: A review and ways forward. Language Teaching Research, 20(6), 741-768. https://doi.org/10.1177/1362168816649979

Macnaughton, D. B. (1998). Which sum of squares are best in an unbalanced analysis of variance? MatStat Research Consulting Inc. https://pdfs.semanticscholar.org/a90a/ddae5a32e69fd174b38e1c9d32ec417a38e4.pdf

Maxwell, S. E., Delaney, H. D., \& Kelley, K. (2018). Designing experiments and analyzing data: A model comparison perspective (3rd ed.). Routledge.

Mayer, A., Dietzfelbinger, L., Rosseel, Y., \& Steyer, R. (2016). The EffectLiteR approach for analyzing average and conditional effects. Multivariate Behavioral Research, 51(2-3), 374-391. https://doi.org/10.1080/00273171.2016.1151334

Mayer, A., \& Thoemmes, F. (2019). Analysis of variance models with stochastic group weights. Multivariate Behavioral Research, 54(4), 542-554. https://doi.org/10.1080/00273171.2018.1548960

McGrath, S., Lin, V., Zhang, Z., Petito, L. C., Logan, R. W., Hernán, M. A., \& Young, J. G. (2020). gfoRmula: An R package for estimating the effects of sustained treatment strategies via the parametric g-formula. Patterns, 1(3), 100008. https://doi.org/10.1016/j.patter.2020.100008

Meyer, D. L. (1991). Misinterpretation of interaction effects: A reply to Rosnow and Rosenthal. Psychological Bulletin, 110(3), 571-573. https://doi.org/10.1037/0033-2909.110.3.571

Naimi, A. I., Cole, S. R., \& Kennedy, E. H. (2016). An introduction to g methods. International Journal of Epidemiology, 46(2), 756-761. https://doi.org/10.1093/ije/dyw323

Navarro, D. J. (2015). Learning statistics with R: A tutorial for psychology students and other beginners (version 0.5). http://health.adelaide.edu.au/psychology/ccs/teaching/lsr/

Nelder, J. A. (1976). Letters to the editor. The American Statistician, 30(2), 103. https://doi.org/10.1080/00031305.1976.10479151 
Nelder, J. A. (1977). A reformulation of linear models. Journal of the Royal Statistical Society. Series A (General), 140(1), 48-77. https://doi.org/10.2307/2344517

Nelder, J. A. (1994). The statistics of linear models: Back to basics. Statistics and Computing, 4(4), 221-234. https://doi.org/10.1007/BF00156745

Nelder, J. A., \& Lane, P. W. (1995). The computer analysis of factorial experiments: In memoriam-Frank Yates. The American Statistician, 49(4), 382-385. https://doi.org/10.1080/00031305.1995.10476189

Neyman, J. (1923/1990). On the application of probability theory to agricultural experiments. Essay on principles. Section 9. Statistical Science, 5(4), 465-472. http://www.jstor.org/stable/2245382

Overall, J. E., Spiegel, D. K., \& Cohen, J. (1975). Equivalence of orthogonal and nonorthogonal analysis of variance. Psychological Bulletin, 82(2), 182-186. https://doi.org/10.1037/h0076381

Overall, J. E., \& Spiegel, D. K. (1969). Concerning least squares analysis of experimental data. Psychological Bulletin, 72(5), 311-322. https://doi.org/10.1037/h0028109

Pearl, J. (2009). Causal inference in statistics: An overview. Statistics Surveys, 3, 96-146. https://doi.org/10.1214/09-SS057

Pedhazur, E. J., \& Schmelkin, L. P. (1991). Measurement, design, and analysis: An integrated approach (Student Edition). Lawrence Erlbaum.

R Core Team. (2020). R: A language and environment for statistical computing (Version 4.0.2) [Computer software]. R Foundation for Statistical Computing. https://www.R-project.org/

Robins, J. (1986). A new approach to causal inference in mortality studies with a sustained exposure period - Application to control of the healthy worker survivor effect. Mathematical Modelling, 7(9), 1393-1512. https://doi.org/10.1016/0270-0255(86)90088-6

Rosenbaum, P. R., \& Rubin, D. B. (1983). The central role of the propensity score in observational studies for causal effects. Biometrika, 70(1), 41-55. https://www.jstor.org/stable/2335942 
Rubin, D. B. (2005). Causal inference using potential outcomes. Journal of the American Statistical Association, 100(469), 322-331. https://doi.org/10.1198/016214504000001880

SAS. (2004). SAS OnlineDoc ${ }^{\circledR}$ (Version 9.1.3) [Computer software]. SAS Institute Inc. https://www.sas.com/en_us/home.html

Searle, S. R. (1987). Linear models for unbalanced data. John Wiley \& Sons.

Shadish, W. R., Cook, T. D., \& Campbell, D. T. (2002). Experimental and quasi-experimental designs for generalized causal inference. Houghton Mifflin.

Shaw, R. G., \& Mitchell-Olds, T. (1993). ANOVA for unbalanced data: An overview. Ecology, 74(6), 1638-1645. https://doi.org/10.2307/1939922

Sigal, M. J., \& Chalmers, R. P. (2016). Play it again: Teaching statistics with monte carlo simulation. Journal of Statistics Education, 24(3), 136-156. https://doi.org/10.1080/10691898.2016.1246953

Skidmore, S. T., \& Thompson, B. (2010). Statistical techniques used in published articles: A historical review of reviews. Educational and Psychological Measurement, 70(5), 777-795. https://doi.org/10.1177/0013164410379320

Speed, F. M., Hocking, R. R., \& Hackney, O. P. (1978). Methods of analysis of linear models with unbalanced data. Journal of the American Statistical Association, 73(361), 105-112. https://doi.org/10.1080/01621459.1978.10480012

Steyer, R., Mayer, A., \& Fiege, C. (2014). Causal inference on total, direct, and indirect effects. In Michalos, A. C. (Ed.), Encyclopedia of Quality of Life and Well-Being Research (pp. 606-631). Springer.

Steyer, R., \& Nagel, W. (2017). Probability and conditional expectation. Fundamentals for the empirical sciences. Wiley.

Steyer, R., Gabler, S., von Davier, A. A., Nachtigall, C., \& Buhl, T. (2000). Causal regression models I: Individual and average causal effects. Methods of Psychological Research Online, 5(2), 39-71. 
Tukey, J. W. (1977). Discussion on Dr Nelder's paper. Journal of the Royal Statistical Society. Series A (General), 140(1), 72. https://doi.org/10.2307/2344517

Venables, W. N. (1998, October 8-9). Exegeses on linear models [Paper presentation]. S-PLUS User's Conference, Washington, DC, United States. http://www.stats.ox.ac.uk/pub/MASS3/Exegeses.pdf

Venables, W. N. (2003, March 7). [r] type iii sum sq in anova table - howto? https://stat.ethz.ch/pipermail/r-help/2003-March/030705.html

Warne, R. T., Lazo, M., Ramos, T., \& Ritter, N. (2012). Statistical methods used in gifted education journals, 2006-2010. Gifted Child Quarterly, 56(3), 134-149. https://doi.org/10.1177/0016986212444122

Wilkinson, G. N., \& Rogers, C. E. (1973). Symbolic description of factorial models for analysis of variance. Applied Statistics, 22(3), 392-399. https://doi.org/10.2307/2346786

Wüthrich-Martone, O. (2001). Causal modeling in psychology with qualitative independent variables. Shaker.

Yates, F. (1934). The analysis of multiple classifications with unequal numbers in the different classes. Journal of the American Statistical Association, 29(185), 51-66. https://doi.org/10.1080/01621459.1934.10502686

Yoo, K., Joo, E., Choi, H., Reid, L., \& Kim, J. (2015). Trends in the use of statistics in major advertising journals over four decades. International Journal of Advertising, 34(3), 549-572. https://doi.org/10.1080/02650487.2015.1005513 